THU-98/32

CERN-TH/98-270

HEP-TH/9808128

August 1998

\title{
U-DUALITY INVARIANCE OF THE Four-Dimensional Born-InfEld Theory
}

\author{
Christiann Hofman $^{1}$, Erik Verlinde ${ }^{1,2}$ and Gysbert Zwart ${ }^{1}$ \\ ${ }^{1}$ Institute for Theoretical Physics, University of Utrecht \\ Princetonplein 5, 3508 TA Utrecht \\ ${ }^{2}$ TH-Division, CERN CH-1211 Geneva 23 \\ hofman@phys.uu.nl, verlinde@phys.uu.nl, zwart@phys.uu.nl
}

\begin{abstract}
We calculate the Hamiltonian of a compactified D4-brane, with general fluxes and moduli, and find the BPS-mass. The results are invariant under the complete U-duality $\mathrm{SO}(5,5, \mathbb{Z})$.
\end{abstract}

\section{INTRODUCTION}

Toroidally compactified string theory is symmetric under a discrete group of U-dualities. These U-dualities act on the various charges present in the theory. In six dimensions, the U-duality group is $\mathrm{SO}(5,5, \mathbb{Z})$; it includes an $\mathrm{SO}(4,4, \mathbb{Z})$-part which is the T-duality group of string theory on a four-torus. The enhancement to the full U-duality group is perhaps better understood from the eleven dimensional point of view; indeed, in [1] the $\mathrm{SO}(5,5, \mathbb{Z})$ symmetry was interpreted as a T-duality of strings living on the M-theory five-brane.

From the ten-dimensional string point of view, the various charges can be split up in NSNS, perturbative ones (momenta and winding) and non-perturbative (RR) charges, 
carried by $\mathrm{D} 0,2$ and 4 -branes wrapping cycles of the four-torus. The $\mathrm{SO}(4,4, \mathbb{Z})$ subgroup respects this split-up, the full U-duality group however mixes both sectors.

The compactified string theory is related to a $4+1$ dimensional gauge theory. The gauge theory is the world volume theory of the D4-branes: a stack of $N$ D4-branes gives rise to a $\mathrm{U}(N)$ gauge theory. Also the other charges are represented in the gauge theory, as electric and magnetic fluxes, compact momenta and the instanton number. Hence a given, generic set of string theory charges can be mapped to a gauge theory configuration with the corresponding set of quantum numbers.

As is well known, the correct action to describe the gauge theory is the Born-Infeld theory [2]. Using this action, we calculate the Hamiltonian as a function of the various fields and moduli. Remarkably, we find a completely $\mathrm{SO}(5,5, \mathbb{Z})$-invariant expression. We also derive a lower bound on the mass given the quantum numbers, the BPS-mass. This mass is identical to the one found in [1] from a six-dimensional space-time supersymmetry calculation.

\section{Dual Pictures}

We are interested in IIA string theory compactified on a four-dimensional torus $\mathbf{T}^{4}$. On this torus we allow for a general configuration of D0-branes, wrapped D4- and D2-branes, winding strings and compact momenta. We want to identify the various conserved charges in this theory and derive the BPS-spectrum, as a function of the moduli. In the end we will demonstrate the invariance of this spectrum under the full U-duality group in six dimensions, $\mathrm{SO}(5,5, \mathbb{Z})$. The system can be looked at from various points of view. The interpretation of both the charges and the moduli depends on the perspective. In the eleven-dimensional point of view the $\mathrm{SO}(5)$ symmetry of the U-duality group is manifest. The zero-brane number is in this picture interpreted as momentum around an additional circle. The quantum numbers are associated to five momenta, 10 membrane-wrapping numbers and the five-brane number. The moduli are unified in a five dimensional metric $G$ and a three-form field $C_{3}$. Together, $G$ and the Hodge dual of $C$ parametrise in the usual fashion the moduli space,

$$
\mathcal{M}_{5,5}=\frac{\mathrm{SO}(5,5)}{\mathrm{SO}(5) \times \mathrm{SO}(5)} .
$$

A dual description is obtained via reduction on $S_{1}$ to get to the string picture. The M-theory five-branes are mapped to D4-branes, which are described by a non-abelian 4+1dimensional Born-Infeld theory. The quantum numbers in this case are the rank of the gauge group, the electric and magnetic fluxes on the four torus, the compact momenta of the gauge theory and the instanton number. The Born-Infeld action contains couplings to the background metric $g, b$-field and RR-forms $c_{1}, c_{3}$, and is multiplied by the string coupling $\lambda$.

The relations between the quantum numbers in the various descriptions of the theory are described in table 1. The sixteen charges transform under the U-duality group $\mathrm{SO}(5,5)$ 


\begin{tabular}{|c|c|c|c|}
\hline quantum \# & strings & $(4+1)$ d Born-Infeld & M-theory \\
\hline$k$ & D0 & instanton number & $P_{5}$ \\
$n_{i}$ & momentum & momentum & $P_{i}$ \\
$w_{i}$ & winding & electric flux & $M_{5 i}$ \\
$m_{i j}$ & D2 & (dual of) magnetic flux & $M_{i j}$ \\
$N$ & D4 & rank & five-brane \\
\hline
\end{tabular}

Table 1: charges in the various pictures

in the sixteen-dimensional spinor representation.

In the following we will derive the BPS-mass from the Born-Infeld action (the D4-branes point of view). We will restrict ourselves to the abelian case, i.e. $N=1$. The resulting expression will then be expressed in terms of the M-theory variables to make its U-duality invariance manifest.

\section{HAMiltonian AND ACTiON}

The abelian Born-Infeld action on the D4-brane is given by

$$
S_{B I}=\int d^{4} x d t \frac{-1}{\lambda} \sqrt{\operatorname{det}(-g-\mathcal{F})}+\frac{1}{2} c_{1} \wedge \mathcal{F} \wedge \mathcal{F}+c_{3} \wedge \mathcal{F},
$$

where $\mathcal{F}=F-b$. Here we use units where the ten-dimensional string length $\ell_{s}=1$. The coupling constant $\lambda$ in front of the first term is the 10 dimensional string coupling; the fact that it appears as $1 / \lambda$ is typical of an RR-soliton. The coupling to the RR potentials $c$ is obtained by expanding $c \wedge \exp \mathcal{F}$.

We want to obtain the Hamiltonian associated to this action, and express it in terms of fields whose zero-modes are the various quantum numbers. These are

$$
\begin{array}{rll}
\text { electric fields } & : E^{i}=\frac{\delta \mathcal{L}}{\delta F_{0 i}}, \\
\text { magnetic fields } & : F_{i j}=\partial_{[i} A_{j]}, \\
\text { momenta } & : P_{i}=F_{i j} E^{j}, \\
\text { instanton density } & : \mathcal{I}=\frac{1}{2} F \wedge F, \\
\text { rank } & : 1 .
\end{array}
$$

Straightforward calculation gives the result

$$
\begin{aligned}
\mathcal{H} & =E^{i} \dot{A}_{i}-\mathcal{L}_{B I} \\
& =\left[\frac{1}{\lambda^{2}}\left(\operatorname{det} g+\frac{\operatorname{det} g}{2}\left(\mathcal{F}_{i j}\right)^{2}+\frac{1}{4}(\mathcal{F} \wedge \mathcal{F})^{2}\right)\right.
\end{aligned}
$$




$$
\left.+\left(\begin{array}{c}
E^{\prime} \\
P^{\prime}
\end{array}\right)^{t}\left(\begin{array}{cc}
g-b g^{-1} b & b g^{-1} \\
-g^{-1} b & g^{-1}
\end{array}\right)\left(\begin{array}{c}
E^{\prime} \\
P^{\prime}
\end{array}\right)\right]^{1 / 2} .
$$

The shifted primed electric field and momentum $E^{\prime}$ and $P^{\prime}$ are given by

$$
E^{\prime}=E-{ }^{*} \mathcal{F} c_{1}+{ }^{*} c_{3}, \quad P^{\prime}=P+\frac{1}{2} \mathcal{F} \wedge \mathcal{F} c_{1}+\mathcal{F}^{*} c_{3} .
$$

We want to rewrite this Hamiltonian such that its U-duality properties become manifest. To this end we first have to group together the different fields in five-dimensional representations, motivated by their M-theory interpretation. The four-momenta $P_{i}$ sit together in a five-dimensional momentum vector with the instanton number density $P_{5} \equiv \frac{1}{2} F \wedge F$; the latter, indeed, is associated to the D0-branes, which are M-theory momentum modes around the extra circle. Similarly, the electric fields are associated to winding strings and therefore are joined to the membrane winding fields ${ }^{*} F$, the four-dimensional hodge dual of the magnetic field. Together they form an antisymmetric tensor,

$$
M_{i j}={ }^{*} F_{i j}, \quad M_{i 5}=E_{i} .
$$

Finally, the rank $N$, the number of five-branes, is a scalar under the $\mathrm{SO}(5)$-rotation group. In the present calculation, $N=1$. In non-abelian gauge theory, the action involves a trace over the group indices, giving $N$ as $\operatorname{Tr} 1$. In the following we will for clarity use $N$ instead of 1 .

In order to change to the M-theory picture, we also upgrade the moduli to fivedimensional ones. The $b$ - and $c_{3}$-fields are components of the eleven-dimensional three-form $C$, whereas the coupling $\lambda$ and the RR 1-form $c_{1}$ come from the 11-dimensional metric,

$$
G=\left(\begin{array}{cc}
g_{i j}+\lambda^{2} c_{1}{ }^{i} c_{1}^{j} & -\lambda^{2} c_{1}^{i} \\
-\lambda^{2} c_{1}^{j} & \lambda^{2}
\end{array}\right),
$$

in string units.

To make the $\mathrm{SO}(5,5) \mathrm{U}$-duality manifest, it is appropriate to convert to six-dimensional Planck units,

$$
\ell_{p l}=\lambda^{\frac{1}{2}}\left(\sqrt{\operatorname{det} g} / \ell_{s}^{4}\right)^{-\frac{1}{4}} \ell_{s}
$$

(see Appendix B), since this is the length scale that is U-invariant. After a rescaling of the metric with a factor $(\operatorname{det} G)^{-1 / 3}$, the Hamiltonian has the form, in units $\ell_{p l}=1$,

$$
\mathcal{H}^{2}=\sqrt{\operatorname{det} G} G^{i j} P_{i}^{\prime} P_{j}^{\prime}+\frac{1}{2 \sqrt{\operatorname{det} G}} G_{i k} G_{j l} M^{\prime i j} M^{\prime k l}+\frac{1}{\sqrt{\operatorname{det} G}} N^{\prime 2},
$$

The three-form field moduli enter in this formula as shifts in the fields, indicated by the primes; this dependence on the three-form $C$, or equivalently its five-dimensional dual two-form ${ }^{*} C$, can be conveniently written, if we identify the vector $P$ with a four-form, as

$$
\begin{aligned}
P^{\prime}+M^{\prime}+N^{\prime} & =e^{-{ }^{*} C} \wedge(P+M+N) \\
& =\left(P-{ }^{*} C \wedge M+\frac{1}{2}{ }^{*} C \wedge{ }^{*} C N\right)+\left(M-{ }^{*} C N\right)+N .
\end{aligned}
$$


If we assume that we can extend the manifest $\mathrm{SL}(4)$ to an $\mathrm{SL}(5)$ action on the fields $P_{i}$ and $M^{i j}$, the Hamiltonian would be manifestly invariant under the $\mathrm{SL}(5, \mathbb{Z})$ coming from the five-torus in M-theory. As we shall see in the next section, this symmetry can even be extended to the full U-duality group, $\mathrm{SO}(5,5, \mathbb{Z})$.

\section{U-DuALITY AND BPS-SPECTRUM}

The Hamiltonian that we have derived from the four-dimensional Born-Infeld action (9) depends on sixteen fields (not all independent) whose zero modes are integer quantum numbers (note that one of these fields is the rank of the gauge group; it is hard to imagine whether it has any modes other than the zero mode). These sixteen quantum numbers, and hence also the fields, sit in the sixteen-dimensional spinor representation of the U-duality group, which is a discrete subgroup of $\mathrm{SO}(5,5)$. The Hamiltonian (9) is invariant under these U-duality transformations. To see how they act it is convenient to represent the fields in terms of a bispinor of $\mathrm{SO}(5)$, satisfying a reality condition; this is equivalent to a spinor of $\mathrm{SO}(5,5)$. We set $\ell_{p l}=1$, and introduce the bispinor

$$
Z=\frac{N^{\prime}}{(\operatorname{det} G)^{1 / 4}}+(\operatorname{det} G)^{1 / 4} P_{i}^{\prime} \Gamma^{i}+\frac{1}{2(\operatorname{det} G)^{1 / 4}} M^{i j} \Gamma_{i j},
$$

where the $\Gamma$ 's are five-dimensional hermitian gamma-matrices satisfying

$$
\left\{\Gamma_{i}, \Gamma_{j}\right\}=2 G_{i j} .
$$

The Hamiltonian then takes the form of the invariant

$$
\mathcal{H}=\frac{1}{2} \sqrt{\operatorname{tr} Z Z^{\dagger}} \equiv\|Z\| .
$$

We now set out to derive a BPS-bound for this system, i.e. a lower bound on the mass given the values of the various quantum numbers. To obtain this bound we introduce the vector [1]

$$
K_{L}=\frac{1}{8} \operatorname{tr} \Gamma Z Z^{\dagger} .
$$

This vector $K_{L}$ can be expressed in terms of the various fields as follows. It is a linear combination of two vectors $K$ and $W$, which in the absence of the ${ }^{*} C$ two-form are given by

$$
K_{i}=N P_{i}-\frac{1}{2}(M \wedge M)_{i}, \quad W^{i}=M^{i j} P_{j}, \quad K_{L i}=K_{i}+G_{i j} W^{j} .
$$

First of all, one can easily check (using the Schouten identity, see Appendix A) that upon inserting the original gauge theory expressions, both vectors are identically zero. If we switch on the ${ }^{*} C$-field, we have to shift $P_{i}$ and $M^{i j}$ :

$$
\begin{aligned}
& P_{i} \rightarrow P_{i}-\left({ }^{*} C \wedge M\right)_{i}+\frac{1}{2}\left({ }^{*} C \wedge{ }^{*} C\right)_{i} N \\
& M \rightarrow M-{ }^{*} C N .
\end{aligned}
$$


Inserting this one immediately finds that $K$ remains unaffected. Using the Schouten identity in five dimensions (see Appendix A), we find

$$
W^{i} \rightarrow W^{i}-{ }^{*} C^{i j} K_{j}
$$

Hence also for general ${ }^{*} C$ the total $K_{L}$ vanishes. (The vanishing of these vectors is precisely equivalent to the fact that the various fields are dependent.)

Using this vector $K_{L}$, we can now derive a BPS-mass formula in terms of the fluxes, which are the zero-modes of the bispinor $Z$, denoted as $Z^{0}$. The details are presented in Appendix 9. The result is

$$
M_{B P S}^{2}=\left\|Z^{0}\right\|^{2}+2\left|K_{L}^{0}\right| .
$$

$K_{L}^{0}$ is defined as the part of $K_{L}$ originating from the zero-modes of $Z$,

$$
K_{L}^{0}=\frac{1}{8} \operatorname{tr} \Gamma Z^{0} Z^{0^{\dagger}}
$$

In components the mass formula reads as follows:

$$
\begin{aligned}
M_{B P S}^{2}= & \sqrt{\operatorname{det} G} G^{-1} n^{\prime 2}+\frac{1}{2} \sqrt{\operatorname{det} G}^{-1} G^{2} m^{\prime 2}+\sqrt{\operatorname{det} G}^{-1} N^{2} \\
& +2 \sqrt{\left(\begin{array}{l}
K^{0} \\
W^{0}
\end{array}\right)\left(\begin{array}{cc}
G^{-1}-{ }^{*} C G^{*} C & { }^{*} C G \\
-G^{*} C & G
\end{array}\right)\left(\begin{array}{l}
K^{0} \\
W^{0}
\end{array}\right)} .
\end{aligned}
$$

The $n^{\prime}, m^{\prime}$ are the zero-modes of the five-dimensional fields, shifted with the terms involving ${ }^{*} C$, as in equation (10). We recognise in the first line the $\operatorname{SO}(5,5)$ invariant constructed from the spinor, in the second line the one from the vector.

Finally, note that if we replace the rank $N$ by a dual five-form field strength, and similarly $m^{\prime}$ by a three-form, the first line in the mass formula attains a more natural form, with just one overall volume factor $\sqrt{\operatorname{det} G}$.

\section{Discussion}

From a matrix theory perspective [3], the theory of $N$ D4-branes describes DLCQ M-theory compactified on a four-torus [4. Therefore, one would expect it to have a duality group $\operatorname{SL}(5, \mathbb{Z})$, the U-duality group in seven dimensions. Indeed, in [5, 6, 7], the masses of the $1 / 2$ BPS-states where derived, and shown to transform in $\operatorname{SL}(5, \mathbb{Z})$ representations. These states correspond in our notation with those having $K_{L}^{0}=0$. It was realised that this group could be enhanced to $\mathrm{SO}(5,5, \mathbb{Z})$ in [0], 8, 9] by adding the generator of Nahm transformations, that exchanges the rank of the gauge group with the fluxes. Furthermore, in [8] degeneracies of a subset of the BPS-states of Yang-Mills theory in three space dimensions were shown to be symmetric under the relevant U-duality group.

In this paper we demonstrated that the full Hamiltonian of the D4-brane, and its BPS mass formula, are symmetric under the U-duality group $\mathrm{SO}(5,5, \mathbb{Z})$, provided one uses the 
full Born-Infeld action. The BPS-spectrum coincides with the one found in [1] from the Mtheory five-brane, which is natural since the D4-brane is the M5-brane wrapped around the eleventh dimension. Just as in that case, we conclude that the D4-brane action is capable of reproducing the correct BPS spectrum of M-theory compactified to six dimensions; a proviso is that the five-brane wrapping number, equal to the rank of the gauge group, be non-vanishing for this description to make sense.

We have seen that the rank $N$ is treated on an equal footing with the fluxes. This suggests that there should be a description of the theory where $N$ is treated as a field, with zero-mode equal to the rank, but also with fluctuations, $N^{\prime}$.

Finally, the procedure carried out in this paper can be easily generalised to dimensions lower than $4+1$, giving the appropriate $\mathrm{E}_{d+1} \mathrm{U}$-duality symmetric mass formulae. In higher dimensions extra quantum numbers corresponding to e.g. NS5-branes, which are not naively present in the gauge theory, have to be added.

\section{ACKNOWLEDGEMENTS}

The authors have benefited from discussions with Feike Hacquebord, Yolanda Lozano, Niels Obers and Boris Pioline. C.H. and G.Z. are supported financially by Stichting FOM. The research of E.V. is partly supported by the Pionier Programme of the Netherlands Organisation for Scientific Research (NWO).

\section{Appendix A. The Schouten Identity}

The Schouten identity states that any 5-rank antisymmetric tensor in 4 dimensions must vanish. It can be written in the form

$$
\varepsilon_{i j k l} \delta_{m}^{n}-\varepsilon_{i j k m} \delta_{l}^{n}-\varepsilon_{i j m l} \delta_{k}^{n}-\varepsilon_{i m k l} \delta_{j}^{n}-\varepsilon_{m j k l} \delta_{i}^{n}=\varepsilon_{[i j k l} \delta_{m]}^{n}=0 .
$$

Contracting this identity with antisymmetric matrices $A_{i j}$ and $B_{k l}$, we find the matrix products

$$
{ }^{*} A B+{ }^{*} B A=-A \wedge B \mathbb{1}
$$

where

$$
{ }^{*} A_{i j}=\frac{1}{2} \varepsilon_{i j k l} A_{k l}, \quad \text { and } \quad A \wedge B=\frac{1}{4} \varepsilon_{i j k l} A_{i j} B_{k l}=\frac{1}{2} * A_{i j} B_{i j} .
$$

In 5 dimensions, there is a similar relation $\varepsilon_{[i j k l m} \delta_{n]}^{p}=0$. Contracting now with three antisymmetric matrices $A, B$ and $C$, we find the relation

$$
A_{i j}(B \wedge C)^{j}+B_{i j}(C \wedge A)^{j}+C_{i j}(A \wedge B)^{j}=0
$$

where

$$
(A \wedge B)^{i}=\frac{1}{4} \varepsilon^{i j k l m} A_{j k} B_{l m} .
$$




\section{ApPendix B. PlanCK Units}

The relation between the 11 -dimensional Planck length $\ell_{p}$ and the string length $\ell_{s}$, is given by

$$
\ell_{p}=\lambda^{1 / 3} \ell_{s}
$$

where $\lambda$ is the string coupling. The Planck length in $11-d$ dimensions, after compactification on a $d$-torus, is given by the formula

$$
\ell_{p l}=\ell_{p}\left(V_{d}\right)^{-1 /(9-d)}
$$

where $V_{d}$ is the volume of the internal $d$-torus in 11-dimensional Planck units. For the case at hand, $d=5$, we find $\ell_{p l}=\ell_{p} \lambda^{1 / 6} v^{-1 / 4}=\lambda^{1 / 2} v^{-1 / 4} \ell_{s}$. The factor $v$ in this expression is the original, four-dimensional volume, $\sqrt{\operatorname{det} g}$, measured in string units $\ell_{s}$. The squared volumes of the five-torus, in the various units, are

$$
\operatorname{det} \frac{G}{\ell_{s}^{2}}=v^{2} \lambda^{2}, \quad \operatorname{det} \frac{G}{\ell_{p}^{2}}=\frac{v^{2}}{\lambda^{\frac{4}{3}}}, \quad \operatorname{det} \frac{G}{\ell_{p l}^{2}}=\frac{v^{\frac{9}{2}}}{\lambda^{3}},
$$

(again with $\ell_{s}^{4} v=\sqrt{\operatorname{det} g}$ ). In our final expression for the Hamiltonian we rescale the metric with a factor $\lambda / v^{3 / 2}$, which equals the determinant in six-dimensional Planck units to the power $-1 / 3$. Therefore, the determinant that appears in equation (9) is

$$
\operatorname{det} G=\left(\frac{\lambda}{v^{3 / 2}}\right)^{5} \frac{v^{\frac{9}{2}}}{\lambda^{3}}=\frac{\lambda^{2}}{v^{3}} .
$$

\section{Appendix C. The BPS-Bound}

The BPS-bound is the minimum of the Hamiltonian, which is derived using a Bogomolny type argument. We introduced the bispinor $Z$, in terms of which the Hamiltonian is defined as

$$
\mathcal{H}=\frac{1}{2} \sqrt{\operatorname{tr} Z Z^{\dagger}} \equiv\|Z\|
$$

Furthermore we need the vector $K_{L}=\frac{1}{8} \operatorname{tr} \Gamma Z Z^{\dagger}$, which is identically zero, as argued in the text. Therefore, we can, for any unit five-vector $e$, write $\mathcal{H}^{2}$ as

$$
\mathcal{H}^{2}=\frac{1}{2}\|Z+e \Gamma Z\|^{2} \equiv \frac{1}{2}\|Z(e)\|^{2} .
$$

The mass of a state is given by the integrated hamiltonian density $\mathcal{H}$,

$$
M=\frac{1}{2} \sqrt{2} \int\|Z(e)\| .
$$


Now we split $Z(e)$ into a piece $Z^{0}(e)$ containing only the zero-modes of the fields, which integrate to the flux quantum numbers, and the non-zero-modes $Z^{\prime}(e)$ (that integrate to zero). Using the inequality

$$
\|Z(e)\| \geq\left\|Z^{0}(e)\right\|+\frac{Z^{0}(e) \cdot Z^{\prime}(e)}{\left\|Z^{0}(e)\right\|},
$$

we obtain the mass-bound

$$
M^{2} \geq \frac{1}{2}\left(\int\left\|Z^{0}(e)\right\|\right)^{2}=\int\left\|Z^{0}\right\|^{2}+2 e \cdot K_{L}^{0}
$$

The BPS-bound can then be obtained be maximising this minimum bound, which evidently leads to

$$
M_{B P S}^{2}=\left\|Z^{0}\right\|^{2}+2\left|K_{L}^{0}\right|
$$

\section{REFERENCES}

[1] R. Dijkgraaf, E. Verlinde and H. Verlinde, BPS Quantization of the Five-Brane, Nucl.Phys. B486 (1997) 89, hep-th/9604055.

[2] R. Leigh, Dirac-Born-Infeld Action from Dirichlet Sigma Models, Mod.Phys.Lett. A4 (1989) 2767.

[3] T. Banks, W. Fischler, S.H. Shenker and L. Susskind, M-Theory as a Matrix Model: A Conjecture, Phys. Rev. D55 (1997) 5112, hep-th/9610043.

[4] W. Taylor IV, D-Brane Field Theory on Compact Spaces, Phys. Lett. B394 (1997) 283, hep-th/9611042. O.J. Ganor, S. Ramgoolam and W. Taylor IV, Branes, Fluxes and Duality in M(atrix) Theory, Nucl. Phys. B492 (1997) 191, hep-th/9611202.

[5] S. Elitzur, A. Giveon, D. Kutasov, E. Rabinovici, Algebraic Aspects of Matrix Theory on $T^{D}$, Nucl. Phys. B509 (1998) 122, hep-th/9707217.

[6] B. Pioline, E. Kiritsis, U-duality and D-brane Combinatorics, Phys Lett. B418 (1998) 61 , hep-th/9710078.

[7] N.A. Obers, B. Pioline, E. Rabinovici, M-Theory and U-duality on $T^{d}$ with Gauge Backgrounds, hep-th/9712084.

[8] F. Hacquebord, H. Verlinde, Duality Symmetry of $\mathcal{N}=4$ Yang-Mills Theory on $T^{3}$, Nucl.Phys. B508 (1997), 609, hep-th/9707179.

[9] M. Blau, M. O'Loughlin, Aspects of U-Duality in Matrix Theory, hep-th/9712047. 